\title{
Biological Function of HYOUI in Tumors and Other Diseases
}

This article was published in the following Dove Press journal: OncoTargets and Therapy

Shan Rao'
Linda Oyang'
Jiaxin Liang'
Pin Yi'
Yaqian Han'
Xia Luo'
Longzheng Xia'
Jinguan Lin '
Shiming Tan'
Jiaqi Hu'
Hui Wang'
Lu Tang ',2
Qing Pan ',2
Yanyan Tang'
Yujuan Zhou'
Qianjin Liao ID '
'Hunan Key Laboratory of Cancer
Metabolism, Hunan Cancer Hospital and
the Affiliated Cancer Hospital of Xiangya
School of Medicine, Central South
University, Changsha, 4 I00I3, Hunan,
People's Republic of China; ${ }^{2}$ University of
South China, Hengyang, 42 I00I, Hunan,
People's Republic of China

Correspondence: Qianjin Liao; Yujuan Zhou

Hunan Key Laboratory of Cancer

Metabolism, Hunan Cancer Hospital and the Affiliated Cancer Hospital of Xiangya School of Medicine, Central South University, 283 Tongzipo Road, Changsha, 4I00I3, Hunan, People's Republic of

China

Tel +86-73I-8865I68I

Fax +86-73I-8865I999

Email march-on@।26.com;

yujany_zhou@163.com

\begin{abstract}
Various stimuli induce an unfolded protein response to endoplasmic reticulum stress, accompanied by the expression of endoplasmic reticulum molecular chaperones. Hypoxia-upregulated 1 gene (HYOU1) is a chaperone protein located in the endoplasmic reticulum. HYOU1 expression was upregulated in many diseases, including various cancers and endoplasmic reticulum stress-related diseases. HYOU1 does not only play an important protective role in the occurrence and development of tumors, but also is a potential therapeutic target for cancer. HYOU1 may also be used as an immune stimulation adjuvant because of its anti-tumor immune response, and a molecular target for therapy of many endoplasmic reticulum-related diseases. In this article, we summarize the updates in HYOU1 and discuss the potential therapeutic effects of HYOU1.
\end{abstract}

Keywords: HYOU1, tumor, endoplasmic reticulum, anti-tumor immunity

\section{Introduction}

The endoplasmic reticulum (ER) is involved in protein synthesis, post-translational modifications, and correct folding of proteins, and plays a key role in many cellular processes. $^{1,2}$ The endoplasmic reticulum is highly sensitive to changes in a range of intracellular environment or extracellular stimuli, especially in malignant cells, including hypoxia, increased protein synthesis, impaired ubiquitination and proteasomal degradation, insufficient energy, nutrition, excessive or restricted substances, and imbalanced calcium levels. ${ }^{3-5}$ These stimuli can lead to accumulation of misfolded proteins in the endoplasmic reticulum cavity, and when the accumulation of misfolded proteins in the endoplasmic reticulum exceeds a certain level, the endoplasmic reticulum will initiate a series of unfolded protein reactions (UPR) to ensure a balance between protein folding capacity and demand. ${ }^{6-10}$ This process initiated by three ER-localized transmembrane proteins-protein kinase-like ER kinase (PERK), inositol-requiring enzyme 1 (IRE1 $\alpha$ ), and activating transcription factor $6 \alpha$ $($ ATF $6 \alpha) .{ }^{11-15}$ The process of endoplasmic reticulum stress is usually accompanied by an increase in endoplasmic reticulum chaperone protein expression, including 78 kDa glucose-regulated protein (GRP78) and Hypoxia-upregulated 1 (HYOU1). ${ }^{16,17}$ Heat shock protein 70 (Hsp70), as a molecular chaperone, is used to maintain and restore the homeostasis of cells. Under stress, Hsp70 has a strong cell protection function. Hsp70 has also been shown to cross-present with major histocompatibility complex class-1 (MHC-1) molecules and has immunomodulatory effects. ${ }^{18-22}$

Hypoxia-upregulated 1 gene (Hyou1) which belongs to the Hsp70 family, also known as $150 \mathrm{kDa}$ oxygen-regulated protein (ORP150). HYOU1 is induced by 
a variety of stimuli, including endoplasmic reticulum stress, hypoxia, ischemia, glucose deficiency, and N-glycosylation inhibitor tunicamycin. ${ }^{23}$ The upregulation of HYOU1 expression is related to many diseases related to endoplasmic reticulum stress, such as diabetes, degenerative neurological diseases and cardiovascular diseases. ${ }^{16,24,25}$ In this review, we outline the latest advances in HYOU1 in human cancer and other diseases, discuss the regulatory mechanisms of HYOU1 in diseases, and discuss possible therapeutic approaches.

\section{Structure and Function of HYOU I}

Hypoxia-upregulated 1 gene (Hyou1), also known as 150 kDa oxygen-regulated protein (ORP150), was first found in rat astrocytes cultured under hypoxic conditions and belongs to the Hsp70 family. ${ }^{26}$ It consists of 999 amino acids encoded by the $3301 \mathrm{bp}$ full-length gene located on the q-arm of chromosome $11 .{ }^{27}$ The sequence of HYOU1 contains a CCAAT-rich cis-acting element called ER stress response element (ERSE), located in the promoter region between intron 1 and exon 2 of the HYOU1 gene. The ERSE element induces HYOU1 during the UPR process by interacting with transcription factors containing consensus sequences. ${ }^{28}$ HYOU1 has a typical HSP70 domain structure, consisting of an N-terminal nucleotide binding domain (NBD), a b-sheet domain, and a C-terminal helix domain. $^{29-31}$

HYOU1 is induced by a variety of stimuli, including endoplasmic reticulum stress, hypoxia, ischemia, glucose deficiency, reducing agent, and tunicamycin. ${ }^{23}$ The upregulation of HYOU1 is related to many diseases related to endoplasmic reticulum stress, such as diabetes, degenerative neurological diseases and cardiovascular diseases. ${ }^{16,24,25}$ HYOU1 plays an important role in crosspresentation, ${ }^{32}$ generates an anti-tumor immune response, and may be used as an immune stimulation adjuvant. ${ }^{30,33}$

The anti-apoptosis and anti-tumor immune response of HYOU1 plays an important role in the occurrence and progress of cancer. ${ }^{29,34,35}$ HYOU1 is involved in the insulin secretion of pancreatic $\beta$ cells. HYOU1 can reduce insulin resistance and improve glucose tolerance in diabetes. ${ }^{36}$ HYOU1 can protect neurons from cell death and plays an important role in neurodegenerative diseases. ${ }^{25,37-39}$ HYOU1 can protect cardiomyocytes and vascular cells from apoptosis, and plays an important role in improving atherosclerosis and regulating cardiac ischemia/reperfusion.

\section{The Role and Mechanism of HYOUI in Tumor Progress}

The supply of glucose, oxygen and other nutrients in the microenvironment of most neoplastic tumors is insufficient, resulting in stress in the endoplasmic reticulum and an increased response to unfolded proteins. Survival chaperones protect tumor cells from ER stress-induced cell death. Studies have shown that many endoplasmic reticulum-resident molecular chaperones, including GRP78, calreticulin, can protect cancer cells from endoplasmic reticulum stress-induced apoptosis. Therefore, the molecular partners of endoplasmic reticulum have become a hot topic in cancer treatment.

HYOU1 is highly expressed in many tumors (Table 1). Many studies have shown that HYOU1 is highly expressed in epithelial ovarian cancer, nasopharyngeal carcinoma, and Kaposi's sarcoma. ${ }^{34,40,41}$ In addition, other studies have

Table I Specific Cancers and Their Association with HYOUI

\begin{tabular}{|l|l|l|l|}
\hline Cancer Type & Expression & Function & Reference \\
\hline Epithelial ovarian cancer & Upregulated & Promoting proliferation, invasion and migration & {$[34]$} \\
\hline Breast cancer & Upregulated & Reducing apoptosis & {$[43]$} \\
\hline Thyroid cancer & Upregulated & Reducing apoptosis & {$[45,52]$} \\
\hline Gastric cancer & Upregulated & Reducing apoptosis & {$[46]$} \\
\hline Bladder cancer & Upregulated & Promoting invasion & {$[42]$} \\
\hline Kaposi's sarcoma & Upregulated & Promoting migration and survival & {$[40]$} \\
\hline Prostate cancer & Upregulated & Promoting angiogenesis and tumorigenicity & {$[44]$} \\
\hline Nasopharyngeal carcinoma & Upregulated & Associating with shorter progression-free survival and overall survival & {$[4 I]$} \\
\hline Non-small cell lung cancer & Upregulated & Promoting invasion, reducing apoptosis, chemoresistance & {$[49]$} \\
\hline
\end{tabular}


shown that HYOU1 expression levels are elevated in breast cancer, bladder cancer, and prostate cancer. ${ }^{42-44}$ HYOU1 is also highly expressed in gastric cancer, thyroid cancer, nonsmall cell lung cancer, and colorectal cancer. ${ }^{45-48}$

\section{Promoting Tumor Progress and the Mechanism}

Studies have shown that the cultured C6 glioma cells showed the common expression of HYOU1 and vascular endothelial growth factor (VEGF). Down-regulation of HYOU1 expression reduced the release of VEGF into the culture supernatant, while overexpression of HYOU1 showed an increase in the level of VEGF antigen, which is tumor growth related. The results show that HYOU1 plays an important role in promoting VEGF secretion and promoting tumor growth. The expression of HYOU1 and VEGF in prostate cancer patients was higher than that of normal people. Inhibiting HYOU1 expression can inhibit VEGF secretion, thereby reducing the angiogenesis and tumorigenicity of prostate cancer DU145 cells. ${ }^{44}$

In human nasopharyngeal carcinoma tissues, the expression of HYOU1 was significantly up-regulated at the mRNA level and protein level, and the expression of HYOU1 protein was positively correlated with the clinical stage and metastasis of NPC. NPC patients with high expression of HYOU1 have shorter progression-free survival (PFS) and overall survival (OS). High expression of HYOU1 was one of the poor prognostic factors in NPC patients. ${ }^{41}$ Proteomic analysis showed elevated HYOU1 expression in non-small cell adenocarcinoma. ${ }^{47}$ Genomic analysis showed that HYOU1 mRNA levels were differentially expressed in colorectal cancer tissues and normal control tissues, and was warranted to further investigate whether it is used as a biomarker for colorectal cancer. ${ }^{48}$

\section{Promoting Tumor Invasion and Migration and the Mechanism}

The level of HYOU1 has also been shown to be associated with cancer invasion. It has been documented that the factors secreted by the crosstalk between endothelial cells (EC) and lung cancer cells lead to the increase of HYOU1 expression in lung cancer spheroids, and the direct interaction between them also leads to the increase of HYOU1 expression in multicellular tumor spheroids (MCTS). Downregulation of HYOU1 can promote cell apoptosis and chemosensitivity in lung cancer MCTS, and inhibit the stemness of lung cancer cells and the expression of EMT-related proteins. HYOU1 also controls tumor growth by regulating IFN signaling in lung cancer cells. In addition, the invasion of lung cancer cells induced by HYOU1 is related to the activation of the $\mathrm{PI} 3 \mathrm{~K} / \mathrm{AKT} / \mathrm{mTOR}$ pathway. Therefore, the selective inhibitor of HYOU1 expression may be used as a promising therapeutic target to overcome the chemoresistance and tumorigenesis of lung cancer. ${ }^{49}$

The expression level of HYOU1 was significantly upregulated in epithelial ovarian cancer tissues and cell lines. Down-regulation of HYOU1 expression can inhibit tumor proliferation and colony formation, as well as ability to migrate and invade. Silencing HYOU1 can down-regulate phosphorylated phosphoinositide 3-kinase (p-PI3K), phosphorylated protein kinase B (p-Akt), and cell cycle and epithelial-mesenchymal transition (EMT) related gene expression levels. High expression of HYOU1 was a poor prognostic factor for overall survival, so that HYOU1 can be used as a target to treat epithelial ovarian cancer. ${ }^{34}$

The expression of HYOU1 is very common in bladder cancer and has a higher expression trend at higher stages. There is a significant correlation between the expression of HYOU1 and metalloproteinase-2 (MMP-2), and HYOU1 acts as a molecular chaperone for MMP-2 secretion to promote tumor invasion. ${ }^{42}$

Kaposi's sarcoma-associated herpes virus (KSHV) vIL-6 can be detected in all malignant tumors related to $\mathrm{KSHV}$, and vIL-6 can promote inflammation and tumorigenesis. vIL-6 mainly exists in the endoplasmic reticulum, and HYOU1 interacts with vIL-6. HYOU1 is highly expressed in Kaposi's sarcoma tissue. HYOU1 increases vIL-6 protein expression and promotes vIL-6-induced janus kinase (JAK)/ signal transducer and activator of transcription (STAT) signaling, migration, and endothelial cell survival. ${ }^{40}$

Studies have evaluated the expression of HYOU1 at various stages of breast cancer, from non-malignant, premalignant and aggressive lesions. They observed differential expression of HYOU1 in breast tissue adjacent to neoplastic lesions and benign tumors, and found a significant correlation between HYOU1 overexpression and poor prognostic histological parameters. Its association with factors, such as regional lymph node metastasis and lymphatic infiltration, suggests that HYOU1 may play a role in metastasis. ${ }^{50}$

\section{The Anti-Apoptotic Role and the Mechanism}

Low glucose-induced HYOU1 expression reduced the apoptosis of breast cancer MCF-7 cells. ${ }^{43}$ 
Proteasome inhibitors have antitumor activity against hematological malignancies and solid tumors. Proteasome inhibitors that interfere with the normal degradation of these proteins are likely to make tumor cells prone to apoptosis. ${ }^{51}$ The study found that the proteasome inhibitor MG132 induced HYOU1 expression in thyroid cancer cells, and its expression was stronger in insensitive thyroid cancer cells than in sensitive cells. Among them, activating transcription factor 4 (ATF4) was involved in the upregulation of HYOU1 induced by MG132. Upregulation of HYOU1 reduced apoptosis by inhibiting the expression of $\mathrm{C} / \mathrm{EBP}$ homologous protein (CHOP), thereby reducing the anti-tumor activity of MG132. ${ }^{45}$ In order to understand the molecular basis of proteasome inhibitor induction of HYOU1 in thyroid cancer, the study found that MG132 induced the expression of HYOU1 via the activation of $-421 /-307$ and $-243 /+53$ regions of HYOU1. Nuclear factor E2-related factor 2 (Nrf2) induced HYOU1 expression in thyroid cancer. Nrf2 directly activated the HYOU1 gene by binding to the $-421 /-307$ region of HYOU1 as a direct transcription activator. Nrf2 also promoted the recruitment of ATF4 to the HYOU1 promoter at $-243 /+53$ activates HYOU1 indirectly. ${ }^{52}$ In human gastric cancer cells, celecoxib upregulated the expression of HYOU1 by inducing an increase in intracellular $\mathrm{Ca} 2+$ concentration, activating the ATF4 and ATF6 pathways, inhibited CHOP expression, and reduced apoptosis, thereby reducing the potential antitumor activity of celecoxib. ${ }^{46}$ Although the research of HYOU1 in tumor cells is extensive, the specific molecular mechanism of action still needs further study (Figure 1).

\section{Role of HYOUI in Anti-Tumor Immunity}

The application of human cancer vaccines has become one of the main goals of basic and clinical cancer research. Studies have confirmed that HSPs such as Hsp70, Hsp90, GRP94 and binding immunoglobulin protein (BiP) can trigger effective tumor-specific cytotoxic $\mathrm{T}$ lymphocyte (CTL) responses. ${ }^{53,54}$ The HSP-Ag complex enters the class I antigen presentation pathway and causes CD8 + $\mathrm{T}$ cell stimulation, which is cross-presentation. ${ }^{55}$ The mechanism of HSP-mediated cross-presentation may be: (1) HSP can promote the main CD8 + T cell response and MHC class I-restricted epitope cross-presentation and (2) HSPs can stimulate innate immune responses that are not related to tumor antigens. The glycosylated form of HYOU1 is used as a potential anti-tumor vaccine.

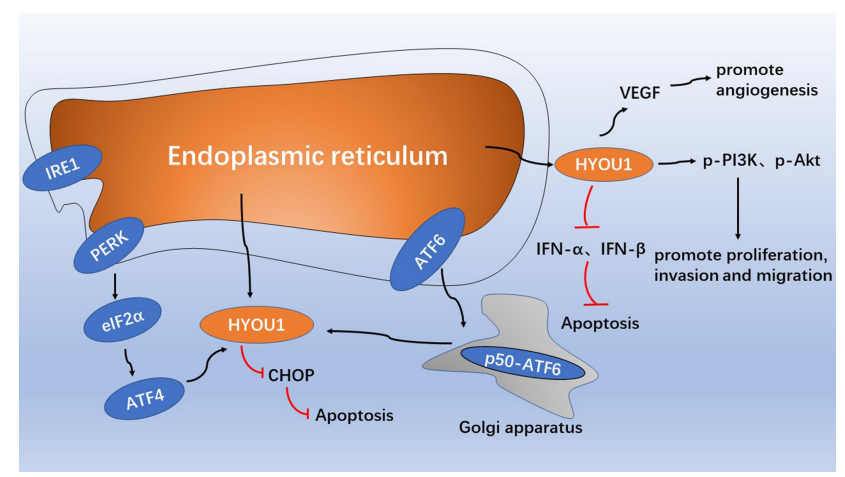

Figure I The role model of intracellular HYOUI in tumor development. The endoplasmic reticulum stress response up-regulates the expression of HYOUI through the PERK-eIF2 $\alpha$-ATF4 pathway and the active form of the p50-ATF6 pathway, and inhibit the expression of CHOP and reduce apoptosis. HYOUI promotes tumor angiogenesis by promoting VEGF secretion. HYOUI promotes cell proliferation, invasion and migration by up-regulating the expression of $\mathrm{p}-\mathrm{PI} 3 \mathrm{~K}$ and $\mathrm{p}-\mathrm{Akt}$. HYOUI reduces apoptosis by down-regulating the expression of IFN- $\alpha$ and IFN- $\beta$.

HYOU1 played an important role in delivering tumor antigens to specialized antigen-presenting cells for crosspresentation, resulting in the generation of anti-tumor immune response that depend on cytotoxic $\mathrm{CD} 8^{+} \mathrm{T}$ cells. HYOU1 can be used as an immunostimulant adjuvant for the development of recombinant vaccines for the treatment of cancer. ${ }^{32}$

Increasing knowledge suggests that prostate stem cell antigen (PSCA) is a promising candidate for immunotherapy of advanced prostate cancer. P. Gao et al had demonstrated that HYOU1 formed a molecular chaperone complex with tumor-associated antigen PSCA during heat shock. The HYOU1-PSCA chaperone complex can enhance $\mathrm{T}$ cell-mediated immune responses, thereby significantly inhibiting tumor growth and prolonging the life span of tumor-bearing mice, which may be an effective method for the treatment of prostate cancer. ${ }^{56}$ Transgenic cancer cells with the ability to produce secret HYOU1 had been successfully tested as a cell-based vaccine that produces a therapeutic anti-tumor response to established tumors in mice $^{56,57}$ The delivery of secreted HYOU1 in tumors by adenovirus can promote the antitumor efficacy of melanoma differentiation-related gene 7 (mda-7), a cancer-specific therapeutic cytokine, by installing a systemic anti-tumor immune system. ${ }^{58}$ Early studies had shown that animals immunized with HYOU1 purified from various murine tumors such as colon tumors, melanomas, and fibrosarcomas had shown a strong anti-tumor immune response. Recombinant chaperone complexes of HYOU1 and melanoma-associated antigen gp100 had been used in the field of oncology. HYOU1 and anti-tumor immunity as a targeted 
vaccine can produce a robust anti-tumor immune response to invasive, poorly immunogenic B16 melanoma in mice. ${ }^{59}$ The above results confirm the important role of tumorderived HYOU1 and Ag complexes associated with GRP tumors in stimulating antigen and tumor-specific immune responses.

HYOU1 can not only be used as an anti-tumor vaccine, but also plays a role in primary immunodeficient diseases. It has been reported that a patient with mutations in HYOU1 is generally sensitive to bacterial and herpes infections and hypoglycemic episodes. ${ }^{60}$

It is implied that HYOU1 may be targeted to develop an effective anti-tumor vaccine, but in-depth research is required to fully explore the anti-cancer potential.

\section{Role of HYOUI in Other Diseases Role of HYOUI in Diabetes}

HYOU1 plays an important role in diabetes. ${ }^{36}$ Studies have found that the expression of HYOU1 is observed in the serum of patients with diabetic nephropathy and is accompanied by an increase in proteinuria and VEGF expression. The results show that HYOU1 is involved in the regulation of proteinuria by mediating VEGF. ${ }^{16}$ In the vitreous samples of patients with proliferative diabetic retinopathy (PDR), a significant increase in VEGF and HYOU1 expression was observed and there was a significant positive correlation between the two. ${ }^{24}$ The study investigated 91 patients with type 1 diabetes and 37 healthy individuals, and measured anti-HYOU1 autoantibody levels in their serum. ${ }^{36}$ They found that serum antiHYOU1 autoantibodies were significantly increased in diabetic patients compared to healthy non-diabetic patients. MIN6 is a known model of insulinoma cell line, HYOU1 has been shown to be highly expressed in mouse MIN6 beta cells under normal oxygen conditions. The study found that the expression of the HYOU1 gene is higher in the insulin resistance library transcription profile than those obtained from insulin-sensitive individuals. Studies on obese diabetic $\mathrm{C} 57 \mathrm{BL} / \mathrm{KsJ}-\mathrm{db} / \mathrm{db}$ and nondiabetic C57BL6 mice have shown that overexpression of HYOU1 reduces insulin resistance in diabetic mice and significantly improves glycemic control. ${ }^{61}$ The increased expression of HYOU1 resulted in a significant increase in the phosphorylation of IRS-1 and Akt and the decrease of expression levels of PEPCK and G6Pase accordingly. ${ }^{61}$ Studies have shown that in the case of type 2 diabetes, HYOU1 can play a role in improving insulin sensitivity of skeletal muscle and liver, but does not participate in insulin secretion. Moreover, systemic overexpression of HYOU1 delayed the onset of disease in homozygous HYOU1/Akita mice and improved insulin sensitivity. On the contrary, the destruction of the HYOU1 gene promotes the development of diabetes and promotes insulin resistance. These findings indicate that ER stress is a key factor in the course of diabetes. Therapies aimed at reducing or enhancing the mechanism of ER stress may become potential treatments for diabetes. As a key mediator of ER stress state, HYOU1 is considered to play an important role in relieving the symptoms of diabetes, but the molecular mechanism and pathway of the specific effect are still not entirely clear.

\section{Role of HYOUI in Neurodegenerative Diseases}

HYOU1 signaling plays an essential role in neurodegenerative diseases. $^{25,37-39}$ The common symptom of many neurodegenerative diseases is the accumulation of misfolded proteins in the lumen of nerve cells, especially Alzheimer's disease and Parkinson's disease. ${ }^{62}$ The study found that in post-mortem brain tissues and cell culture models, upregulation of ER stress markers was observed in diseases such as Alzheimer's disease and Parkinson's disease. Studies have also shown that endoplasmic reticulum stress and endoplasmic reticulum partner molecules have a direct role in the pathogenesis of Alzheimer's disease. ${ }^{63,64}$ HYOU1 is involved in maintaining neuronal cell homeostasis and the survival of vulnerable neurons, and is susceptible to excitatory stimulation. ${ }^{65-67}$ The expression of HYOU1 has been identified in the human hippocampus in human brain tissue after alginate injection after seizures. In addition, the up-regulation of HYOU1 expression in the gerbil brain inhibited the delayed death of nerve cells after ischemia, indicating its cytoprotective effect. ${ }^{68}$ Studies had found that $A \beta 42$ in Alzheimer's disease can be prevented by inhibiting HYOU1 or activating the E3-ligase activity of CHIP. ${ }^{37}$ Over-expression of HYOU1 under stress can protect neurons from cell death. The data indicate that endoplasmic reticulum stress may be the main cause of neuronal cell death, and HYOU1 may become a promising neuroprotective mediator. ${ }^{65,69,70}$ However, in-depth research is still needed to fully clarify the role and exact therapeutic potential of HYOU1 in neurodegenerative diseases. 


\section{Role of HYOUI in Cardiovascular Diseases}

The study found that HYOU1 autoantibodies have been detected in the serum of patients with severe atherosclerosis. Oxidized low density lipoproteins (oxLDLs) are known to induce apoptosis and promote atherosclerosis. OxLDL enhances the expression of HYOU1 in cultured vascular endothelial cells and smooth muscle cells, and HYOU1 protects endothelial cells from oxLDL-induced apoptosis. Studies have also shown that after incubation of HMEC-1 cells with oxLDL, ER stress markers and ER chaperone proteins GRP78, GRP94 and HYOU1 are clearly expressed. Down-regulation of HYOU1 enhances caspase-3 activation and oxLDL-induced cytotoxicity, while overexpression of HYOU1 in HMEC cells reduces oxLDL-induced caspase-3 activation and IRE1 and c-Jun N-terminal kinase (JNK) phosphorylation. In addition, the down-regulation of HYOU1 leads to inhibition of Akt phosphorylation. ${ }^{71}$ Studies have also shown that the antiER stress and anti-apoptotic effects of HYOU1 occur downstream of AMPK signaling in liver cell lines. The enhanced expression of HYOU1 plays an important role in preventing apoptosis events of macrophages, vascular cells and cardiomyocytes.

\section{Conclusion}

HYOU1 is an endoplasmic reticulum chaperone protein, belonging to the HSP70 family, induced by a variety of stress conditions, such as hypoxia, glucose deficiency, reducing agents and tunicamycin. In general, HYOU1 inhibits apoptosis, maintains calcium homeostasis, and exhibits cytoprotective effects by preventing endoplasmic reticulum stress. ${ }^{29}$ The anti-apoptotic effects of HYOU1 include: (1) Attenuation of ER stress-related apoptosis pathways, such as reduced phosphorylation of IRE1 and JNK and downregulation of XBP1, XBP1s and CHOP expression, (2) through HYOU1 and ER stress sensor PERK, the possible combination of ATF6 and IRE1 keeps them inactive, thereby inhibiting UPR, and (3) activation of Akt phosphorylation as part of the survival phosphatidylinositol 3-kinase/Akt signal transduction pathway. ${ }^{27}$ Preliminary studies indicate that HYOU1 directly binds to PERK, ATF6 and IRE1. The results show that GRP78 may not be the only molecule that binds to the UPR sensor, and HYOU1 may be another protein that can bind to the UPR sensor receptor to inhibit or reduce the response of unfolded proteins, but further indepth analysis and research are needed to solve this problem. Although HYOU1 has been the topic of extensive research since its emergence, its molecular mode of action in cancer biology is still unclear. It is necessary to further explore its specific mechanisms of action and further explore the potential of HYOU1 in clinical treatment.

\section{Abbreviations}

HYOU1, hypoxia-upregulated 1; ER, the endoplasmic reticulum; PERK, proteins-protein kinase-like ER kinase; IRE1 $\alpha$, inositol-requiring enzyme 1; ATF6 $\alpha$, activating transcription factor $6 \alpha$; GRP78, $78 \mathrm{kDa}$ glucose-regulated protein; Hsp70, heat shock protein 70; MHC-1, major histocompatibility complex class-1; ORP150, $150 \mathrm{kDa}$ oxygen-regulated protein; ERSE, ER stress response element; NBD, N-terminal nucleotide binding domain; VEGF, vascular endothelial growth factor; PFS, progression-free survival; OS, overall survival; p-PI3K, phosphorylated phosphoinositide 3-kinase; p-Akt, phosphorylated protein kinase B; EMT, epithelial-mesenchymal transition; MMP-2, metalloproteinase-2; KSHV, Kaposi's sarcoma-associated herpes virus; JAK, janus kinase; STAT, signal transducer and activator of transcription.

\section{Author Contributions}

All authors made a significant contribution to the work reported, whether that is in the conception, study design, execution, acquisition of data, analysis and interpretation, or in all these areas; took part in drafting, revising or critically reviewing the article; gave final approval of the version to be published; have agreed on the journal to which the article has been submitted; and agree to be accountable for all aspects of the work.

\section{Funding}

This work was supported in part by grants from the following sources: the National Natural Science Foundation of China $(81972636,81872281,81772842)$, the Natural Science Foundation of Hunan Province (2020JJ5336, 2019JJ40175, 2019JJ40183 2018JJ1013), the Research Project of Health Commission of Hunan Province (202109031837, 20201020), China Hunan Provincial Science and Technology Department (2018SK7005), Ascend Foundation of National cancer center (NCC2018b68), and Supported By Hunan Cancer Hospital Climb Plan (ZX2020001-3, YF2020002) and By the Fundamental Research Funds for the Central Universities of Central South University (2019zzts832, 2019zzts833). 


\section{Disclosure}

The authors declare that they have no competing interests.

\section{References}

1. Yang Y, Zhou Q, Gao A, Chen L, Li L. Endoplasmic reticulum stress and focused drug discovery in cardiovascular disease. Clin Chim Acta. 2020;504:125-137. doi:10.1016/j.cca.2020.01.031

2. Oakes SA. Endoplasmic reticulum stress signaling in cancer cells. Am J Pathol. 2020;190(5):934-946. doi:10.1016/j.ajpath.2020.01.010

3. Mou Z, Yuan YH, Zhang Z, Song LK, Chen NH. Endoplasmic reticulum stress, an important factor in the development of Parkinson's disease. Toxicol Lett. 2020;324:20-29. doi:10.1016/j. toxlet.2020.01.019

4. Di Conza G, Ho PC. ER stress responses: an emerging modulator for innate immunity. Cells. 2020;9(3):695. doi:10.3390/cells9030695

5. da Silva DC, Valentao P, Andrade PB, Pereira DM. Endoplasmic reticulum stress signaling in cancer and neurodegenerative disorders: tools and strategies to understand its complexity. Pharmacol Res. 2020;155:104702.

6. Liu X, Liu J. Tanshinone I induces cell apoptosis by reactive oxygen species-mediated endoplasmic reticulum stress and by suppressing p53/DRAM-mediated autophagy in human hepatocellular carcinoma. Artif Cells Nanomed Biotechnol. 2020;48(1):488-497.

7. Lorenzon-Ojea AR, Yung HW, Burton GJ, Bevilacqua E. The potential contribution of stromal cell-derived factor 2 (SDF2) in endoplasmic reticulum stress response in severe preeclampsia and labor-onset. Biochim Biophys Acta Mol Basis Dis. 2020;1866(2):165386. doi:10.1016/j.bbadis.2019.01.012

8. Losada A, Berlanga JJ, Molina-Guijarro JM, et al. Generation of endoplasmic reticulum stress and inhibition of autophagy by plitidepsin induces proteotoxic apoptosis in cancer cells. Biochem Pharmacol. 2020;172:113744.

9. Ma YY, Di ZM, Cao Q, et al. Xanthatin induces glioma cell apoptosis and inhibits tumor growth via activating endoplasmic reticulum stress-dependent CHOP pathway. Acta Pharmacol Sin. 2020;41 (3):404-414.

10. Mao Y, Wang C, Tian X, et al. Endoplasmic reticulum stress contributes to nociception via neuroinflammation in a murine bone cancer pain model. Anesthesiology. 2020;132(2):357-372. doi:10. 1097/ALN.0000000000003078

11. Pan X, Cao YM, Liu JH, Ding J, Xie XY, Cao P-G. MEG3 induces cervical carcinoma cells' apoptosis through endoplasmic reticulum stress by miR-7-5p/STC1 axis. Cancer Biother Radiopharm. 2020. doi: $10.1089 /$ cbr.2019.3344

12. Pibiri M, Sulas P, Camboni T, Leoni VP, Simbula G. Alpha-lipoic acid induces endoplasmic reticulum stress-mediated apoptosis in hepatoma cells. Sci Rep. 2020;10(1):7139. doi:10.1038/s41598-02064004-5

13. Qin XY, Su T, Yu W, Kojima S. Lipid desaturation-associated endoplasmic reticulum stress regulates MYCN gene expression in hepatocellular carcinoma cells. Cell Death Dis. 2020;11(1):66. doi:10. 1038/s41419-020-2257-y

14. Riaz TA, Junjappa RP, Handigund M, Ferdous J, Kim HR, Chae HJ. Role of endoplasmic reticulum stress sensor irelalpha in cellular physiology, calcium, ROS signaling, and metaflammation. Cells. 2020;9(5):1160. doi:10.3390/cells9051160

15. Rodvold JJ, Xian S, Nussbacher J, et al. IRE1alpha and IGF signaling predict resistance to an endoplasmic reticulum stress-inducing drug in glioblastoma cells. Sci Rep. 2020;10(1):8348. doi:10.1038/s41598020-65320-6

16. Wang DQ, Miao XJ, Gao J, Zhou YH, Ji FY, Cheng XB. The $150-\mathrm{kDa}$ oxygen-regulated protein (ORP150) regulates proteinuria in diabetic nephropathy via mediating VEGF. Exp Mol Pathol. 2019;110:104255. doi:10.1016/j.yexmp.2019.04.014
17. Li Y, Sun M, Liu Y, Liang J, Wang T, Zhang Z. Gymnemic acid alleviates type 2 diabetes mellitus and suppresses endoplasmic reticulum stress in vivo and in vitro. J Agric Food Chem. 2019;67 (13):3662-3669. doi:10.1021/acs.jafc.9b00431

18. Vostakolaei MA, Abdolalizadeh J, Hejazi MS, Kordi S, Molavi O. Hsp70 in cancer: partner or traitor to immune system. Iran J Allergy Asthma Immunol. 2019;18(6):589-604. doi:10.18502/ijaai.v18i6.2172

19. Rosenzweig R, Nillegoda NB, Mayer MP, Bukau B. The Hsp70 chaperone network. Nat Rev Mol Cell Biol. 2019;20(11):665-680. doi:10.1038/s41580-019-0133-3

20. Moran Luengo T, Mayer MP, Rudiger SGD. The Hsp70-Hsp90 chaperone cascade in protein folding. Trends Cell Biol. 2019;29 (2):164-177. doi:10.1016/j.tcb.2018.10.004

21. Genest O, Wickner S, Doyle SM. Hsp90 and Hsp70 chaperones: collaborators in protein remodeling. J Biol Chem. 2019;294 (6):2109-2120. doi:10.1074/jbc.REV118.002806

22. Clerico EM, Meng W, Pozhidaeva A, Bhasne K, Petridis C, Gierasch LM. Hsp70 molecular chaperones: multifunctional allosteric holding and unfolding machines. Biochem J. 2019;476 (11):1653-1677. doi:10.1042/BCJ20170380

23. Kuwabara K, Matsumoto M, Ikeda J, et al. Purification and characterization of a novel stress protein, the $150-\mathrm{kDa}$ oxygen-regulated protein (ORP150), from cultured rat astrocytes and its expression in ischemic mouse brain. J Biol Chem. 1996;271(9):5025-5032. doi:10.1074/jbc. 271.9 .5025

24. Abu El-Asrar AM, Ahmad A, Alam K, et al. Association of 150-kDa oxygen-regulated protein with vascular endothelial growth factor in proliferative diabetic retinopathy. Acta Ophthalmol. 2018;96(4): e460-e467. doi:10.1111/aos.13600

25. Grande V, Ornaghi F, Comerio L, et al. PERK inhibition delays neurodegeneration and improves motor function in a mouse model of Marinesco-Sjogren syndrome. Hum Mol Genet. 2018;27 (14):2477-2489. doi:10.1093/hmg/ddy 152

26. Tsukamoto Y, Kuwabara K, Hirota S, et al. 150-kD oxygen-regulated protein is expressed in human atherosclerotic plaques and allows mononuclear phagocytes to withstand cellular stress on exposure to hypoxia and modified low density lipoprotein. J Clin Invest. 1996;98 (8):1930-1941. doi:10.1172/JCI118994

27. Kusaczuk M, Cechowska-Pasko M. Molecular chaperone ORP150 in ER stress-related diseases. Curr Pharm Des. 2013;19(15):28 07-2818. doi:10.2174/1381612811319150016

28. Takeuchi S. Molecular cloning, sequence, function and structural basis of human heart $150 \mathrm{kDa}$ oxygen-regulated protein, an ER chaperone. Protein J. 2006;25(7-8):517-528. doi:10.1007/s10930006-9038-z

29. Pagare PP, Wang H, Wang XY, Zhang Y. Understanding the role of glucose regulated protein 170 (GRP170) as a nucleotide exchange factor through molecular simulations. $J$ Mol Graph Model. 2018;85:160-170. doi:10.1016/j.jmgm.2018.09.001

30. Bracher A, Verghese J. The nucleotide exchange factors of Hsp70 molecular chaperones. Front Mol Biosci. 2015;2:10. doi:10.3389/ fmolb.2015.00010

31. Behnke J, Mann MJ, Scruggs FL, Feige MJ, Hendershot LM. Members of the Hsp70 family recognize distinct types of sequences to execute ER quality control. Mol Cell. 2016;63(5):739-752. doi:10.1016/j.molcel.2016.07.012

32. Wang H, Pezeshki AM, Yu X, Guo C, Subjeck JR, Wang XY. The endoplasmic reticulum chaperone GRP170: from immunobiology to cancer therapeutics. Front Oncol. 2014;4:377. doi:10.3389/fonc.2014.00377

33. Zuo D, Subjeck J, Wang XY. Unfolding the role of large heat shock proteins: new insights and therapeutic implications. Front Immunol. 2016;7:75. doi:10.3389/fimmu.2016.00075

34. Li X, Zhang NX, Ye HY, et al. HYOU1 promotes cell growth and metastasis via activating PI3K/AKT signaling in epithelial ovarian cancer and predicts poor prognosis. Eur Rev Med Pharmacol Sci. 2019;23(10):4126-4135. doi:10.26355/eurrev_201901_17914 
35. Inoue T, Tsai B, Gilmore R. The Grp170 nucleotide exchange factor executes a key role during ERAD of cellular misfolded clients. Mol Biol Cell. 2016;27(10):1650-1662. doi:10.1091/mbc.E16-01-0033

36. Nakatani Y, Kaneto H, Hatazaki M, et al. Increased stress protein ORP150 autoantibody production in Type 1 diabetic patients. Diabetic Med. 2006;23(2):216-219. doi:10.1111/j.1464-5491.2005. 01766.x

37. Chanana N, Pati U. ORP150-CHIP chaperone antagonism control BACE1-mediated amyloid processing. J Cell Biochem. 2018;119 (6):4615-4626. doi:10.1002/jcb.26630

38. Ahmadinejad F, Geir Moller S, Hashemzadeh-Chaleshtori M, Bidkhori G, Jami MS. Molecular mechanisms behind free radical scavengers function against oxidative stress. Antioxidants (Basel). 2017;6(3):3. doi:10.3390/antiox6030051

39. Zhao L, Rosales C, Seburn K, Ron D, Ackerman SL. Alteration of the unfolded protein response modifies neurodegeneration in a mouse model of Marinesco-Sjogren syndrome. Hum Mol Genet. 2010;19 (1):25-35. doi:10.1093/hmg/ddp464

40. Giffin L, Yan F, Ben Major M, Damania B. Modulation of Kaposi's sarcoma-associated herpesvirus interleukin- 6 function by hypoxiaupregulated protein 1. J Virol. 2014;88(16):9429-9441. doi:10.1128/ JVI.00511-14

41. Zhou Y, Liao Q, Li X, et al. HYOU1, regulated by LPLUNC1, is up-regulated in nasopharyngeal carcinoma and associated with poor prognosis. J Cancer. 2016;7(4):367-376. doi:10.7150/jca.13695

42. Asahi H, Koshida K, Hori O, Ogawa S, Namiki M. Immunohistochemical detection of the $150-\mathrm{kDa}$ oxygen-regulated protein in bladder cancer. BJU Int. 2002;90(4):462-466. doi:10.10 46/j.1464-410X.2002.02915.x

43. Kretowski R, Borzym-Kluczyk M, Stypulkowska A, BranskaJanuszewska J, Ostrowska H, Cechowska-Pasko M. Low glucose dependent decrease of apoptosis and induction of autophagy in breast cancer MCF-7 cells. Mol Cell Biochem. 2016;417(1-2):35-47. doi:10.1007/s11010-016-2711-4

44. Miyagi T, Hori O, Koshida K, et al. Antitumor effect of reduction of $150-\mathrm{kDa}$ oxygen-regulated protein expression on human prostate cancer cells. Int J Urol. 2002;9(10):577-585. doi:10.1046/j.14422042.2002.00519.x

45. Gao YY, Liu BQ, Du ZX, Zhang HY, Niu XF, Wang HQ. Implication of oxygen-regulated protein 150 (ORP150) in apoptosis induced by proteasome inhibitors in human thyroid cancer cells. $J$ Clin Endocrinol Metab. 2010;95(11):E319-326. doi:10.1210/jc.2010-1043

46. Namba T, Hoshino T, Tanaka K, et al. Up-regulation of $150-\mathrm{kDa}$ oxygen-regulated protein by celecoxib in human gastric carcinoma cells. Mol Pharmacol. 2007;71(3):860-870. doi:10.1124/mol.106.027698

47. Fahrmann JF, Grapov D, Phinney BS, et al. Proteomic profiling of lung adenocarcinoma indicates heightened DNA repair, antioxidant mechanisms and identifies LASP1 as a potential negative predictor of survival. Clin Proteomics. 2016;13:31. doi:10.1186/s12014-016-91 $32-\mathrm{y}$

48. Zhang J, Yan B, Spath SS, et al. Integrated transcriptional profiling and genomic analyses reveal RPN2 and HMGB1 as promising biomarkers in colorectal cancer. Cell Biosci. 2015;5(1):53. doi:10.1186/ s13578-015-0043-9

49. Lee M, Song Y, Choi I, et al. Expression of HYOU1 via reciprocal crosstalk between NSCLC Cells and HUVECs control cancer progression and chemoresistance in tumor spheroids. Mol Cells. 2021;44 (1):50-62. doi:10.14348/molcells.2020.0212

50. Stojadinovic A, Hooke JA, Shriver CD, et al. HYOU1/Orp150 expression in breast cancer. Med Sci Monit. 2007;13(11):BR231-239.

51. Adams J. The proteasome: a suitable antineoplastic target. Nat Rev Cancer. 2004;4(5):349-360. doi:10.1038/nrc1361

52. Zong ZH, Du ZX, Zhang HY, et al. Involvement of Nrf2 in proteasome inhibition-mediated induction of ORP150 in thyroid cancer cells. Oncotarget. 2016;7(3):3416-3426. doi:10.18632/oncotarget. 6636
53. Sasiain MC, de la Barrera S, Fink $\mathrm{S}$, et al. Interferon-gamma (IFN-gamma) and tumour necrosis factor-alpha (TNF-alpha) are necessary in the early stages of induction of CD4 and CD8 cytotoxic $\mathrm{T}$ cells by Mycobacterium leprae heat shock protein (hsp) $65 \mathrm{kD}$. Clin Exp Immunol. 1998;114(2):196-203. doi:10.1046/j.1365-2249.1998. 00702.x

54. Ghio M, Fabbi P, Contini P, Fedele M, Brunelli C, Indiveri F. OxLDL- and HSP-60 antigen-specific CD8(+) T lymphocytes are detectable in the peripheral blood of patients suffering from coronary artery disease. Clin Exp Med. 2013;13(4):251-255. doi:10.1007/ s10238-012-0205-6

55. Santos GJL, Oliveira ES, Pinheiro ADN, et al. Himatanthus drasticus (Apocynaceae) latex reduces oxidative stress and modulates CD4(+), CD8(+), FoxP3(+) and HSP-60(+) expressions in Sarcoma 180-bearing mice. J Ethnopharmacol. 2018;220:159-168. doi:10.11 58/0008-5472.CAN-05-2609

56. Gao P, Sun X, Chen X, Subjeck J, Wang X-Y. Secretion of stress protein grp170 promotes immune-mediated inhibition of murine prostate tumor. Cancer Immunol Immunother. 2009;58(8):13 19-1328. doi:10.1007/s00262-008-0647-6

57. Wang X-Y, Arnouk H, Chen X, Kazim L, Repasky EA, Subjeck JR. Extracellular targeting of endoplasmic reticulum chaperone glucose-regulated protein 170 enhances tumor immunity to a poorly immunogenic melanoma. J Immunol. 2006;177(3):1543-1551. doi:10.4049/jimmunol.177.3.1543

58. Gao P, Sun X, Chen X, et al. Secretable chaperone Grp170 enhances therapeutic activity of a novel tumor suppressor, mda-7/IL-24. Cancer Res. 2008;68(10):3890-3898.

59. Park JE, Facciponte J, Chen X, et al. Chaperoning function of stress protein grp170, a member of the hsp70 superfamily, is responsible for its immunoadjuvant activity. Cancer Res. 2006;66(2):1161-1168.

60. Haapaniemi EM, Fogarty CL, Keskitalo S, et al. Combined immunodeficiency and hypoglycemia associated with mutations in hypoxia upregulated 1. J Allergy Clin Immunol. 2017;139(4):1391-1393e 1311. doi:10.1016/j.jaci.2016.09.050

61. Ozawa K, Kondo T, Hori O, et al. Expression of the oxygen-regulated protein ORP150 accelerates wound healing by modulating intracellular VEGF transport. J Clin Invest. 2001;108(1):41-50. doi:10.1172/ JCI200111772

62. Lindholm D, Wootz H, Korhonen L. ER stress and neurodegenerative diseases. Cell Death Differ. 2006;13(3):385-392. doi:10.1038/sj. cdd. 4401778

63. Endres K, Reinhardt S. ER-stress in Alzheimer's disease: turning the scale? Am J Neurodegener Dis. 2013;2(4):247-265.

64. de la Monte SM, Re E, Longato L, Tong M. Dysfunctional proceramide, ER stress, and insulin/IGF signaling networks with progression of Alzheimer's disease. J Alzheimers Dis. 2012;30(Suppl 2): S217-229. doi:10.3233/JAD-2012-111728

65. Buchanan H, Mackay M, Palmer K, Tothova K, Katsur M, Platt B. Synaptic loss, ER stress and neuro-inflammation emerge late in the lateral temporal cortex and associate with progressive tau pathology in Alzheimer's disease. Mol Neurobiol. 2020;57(8):3258-3272. doi:10.1007/s12035-020-01950-1

66. Sadleir KR, Popovic J, Vassar R. ER stress is not elevated in the 5XFAD mouse model of Alzheimer's disease. J Biol Chem. 2018;293 (48):18434-18443. doi:10.1074/jbc.RA118.005769

67. Gerakis Y, Hetz C. Emerging roles of ER stress in the etiology and pathogenesis of Alzheimer's disease. FEBS J. 2018;285(6):9 95-1011. doi:10.1111/febs. 14332

68. Kitao Y, Ozawa K, Miyazaki M, et al. Expression of the endoplasmic reticulum molecular chaperone (ORP150) rescues hippocampal neurons from glutamate toxicity. J Clin Invest. 2001;108(10):1439-1450. doi: $10.1172 / \mathrm{JCI} 12978$

69. Uddin MS, Tewari D, Sharma G, et al. Molecular mechanisms of ER stress and UPR in the pathogenesis of Alzheimer's disease. Mol Neurobiol. 2020;57(7):2902-2919. 
70. Salminen A, Kaarniranta K, Kauppinen A. ER stress activates immunosuppressive network: implications for aging and Alzheimer's disease. J Mol Med (Berl). 2020;98(5):633-650. doi:10.1007/s00 109-020-01904-Z
71. Sanson M, Ingueneau C, Vindis $C$, et al. Oxygen-regulated protein-150 prevents calcium homeostasis deregulation and apoptosis induced by oxidized LDL in vascular cells. Cell Death Differ. 2008;15(8):1255-1265.

\section{Publish your work in this journal}

OncoTargets and Therapy is an international, peer-reviewed, open access journal focusing on the pathological basis of all cancers, potential targets for therapy and treatment protocols employed to improve the management of cancer patients. The journal also focuses on the impact of management programs and new therapeutic

Submit your manuscript here: https://www.dovepress.com/oncotargets-and-therapy-journal agents and protocols on patient perspectives such as quality of life, adherence and satisfaction. The manuscript management system is completely online and includes a very quick and fair peer-review system, which is all easy to use. Visit http://www.dovepress.com/ testimonials.php to read real quotes from published authors. 